\title{
Highly Sensitive Luminescent Probe for Singlet Oxygen Based on A Europium (III) Complexes Containing Benzoyltrifluoroacetone and (1-(Anthracen-9-yl)-N-(pyridine- 2-yl methyl) Methaneamine Ligands
}

\author{
Dinh Thi Hien ${ }^{*}$, Pham Thi Nhan \\ Hanoi National University of Education, 136 Xuan Thuy, Cau Giay, Hanoi, Vietnam
}

Received 01 April 2020

Revised 08 April 2020; Accepted 09 March 2021

\begin{abstract}
A new luminescent probe based on $\beta$ - diketonate $\mathrm{Eu}^{3+}$ complex, Eu (TFPB) ${ }_{3} \mathrm{AnPy}$, where AnPy is (1-(anthracen-9-yl)-N-(pyridine-2-ylmethyl) methaneamine and TFPB is benzoyltriluoro acetone was successfully synthesized. The molecular structure of Eu (TFPB) ${ }_{3}$ AnPy determined by SC-XRD show an eight coordination in which Eu3+ is bonded to six oxygen atoms of three TFPB and two nitrogen atoms of the AnPy ligand. The complex could act as a highly sensitive luminescent probe for ${ }^{1} \mathrm{O}_{2}$ that can be applied in biological systems.
\end{abstract}

Keywords: Rare earth complex, $\beta$-diketonate, anthracene.

\section{Introduction}

Singlet oxygen $\left({ }^{1} \mathrm{O}_{2}\right)$, the excited state of molecular oxygen, is an important reactive species and a strong oxidant that has been widely used in chemistry and biochemistry. In the biological systems, ${ }^{1} \mathrm{O}_{2}$ is considered to be an important toxic species since it can oxidize various biological molecules such as DNA, proteins, and lipids $[1,3,5]$. Due to the significant importance of ${ }^{1} \mathrm{O}_{2}$ in photobiological and photochemical processes, the stable and highly selective and sensitive probes for ${ }^{1} \mathrm{O}_{2}$

\footnotetext{
* Corresponding Author.

Email address: dth0104@gmail.com
}

https://doi.org/10.25073/2588-1140/vnunst.4992 have attracted much interest. Several methods for detecting ${ }^{1} \mathrm{O}_{2}$ have been developed [1,3]. Some luminescent probe singlet oxygen based on a $\beta$-diketonate-europium complex was successfully designed and synthesized $[2,4]$. The main benefits of this probe are facile synthesis and enhanced emission quantum yield. As a result, a large number of luminescent $\beta$-diketonate- $\mathrm{Eu}^{3+}$ complexes can be used to detect singlet oxygen in living biosystems. In this work, by using an anthryl substituted amine ligand (1-(anthracen-9-yl)-N(pyridine-2-ylmethyl)methaneamine (AnPy) and a $\beta$-diketone coligand benzoyltrifluoroacetone (TFPB), a new $\beta$ - diketonate - $\mathrm{Eu}^{3+}$ complex- based luminescent probe, $\mathrm{Eu}$ (TFPB $)_{3}$ AnPy was synthesized. The probe was 
weakly luminescent due to the strong quenching of 9-anthryl group to the $\mathrm{Eu}^{3+}$ emission. After the specific reaction of 9anthryl group with ${ }^{1} \mathrm{O}_{2}$, the endoperoxide derivative of the probe, $\mathrm{Eu}(\mathrm{TFPB})_{3}(\mathrm{EP}-\mathrm{AnPy})$ was formed, followed by increase in luminescence intensity.

\section{Experimental}

\subsection{Materials and instruments}

All the solvents used for synthesis and spectroscopic measurements were purified according to literature procedures.

The IR spectra of M2 was measured using a FT-IR 8700 infrared spectrophotometer (4000 - $400 \mathrm{~cm}^{-1}$ ) in $\mathrm{KBr}$ pellets at Institute of Chemistry, Vietnam Academy of Science and Technology.

Single crystal $X$-ray diffraction data of the complex M2 was measured on the X-ray diffractometer (Bruker D8 Quest) at 298K at the Faculty of Chemistry, Hanoi National University. Structure solution and refinement were performed using OLEX2 programs.

Absorption spectra of the ligands and the complexes were measured in dichloromethane at room temperature on Cary $5000 \mathrm{UV} / \mathrm{Vis}$ spectrometer at Faculty of Environmental Chemistry, Hanoi National University of Education.

Photoluminescence spectroscopy of Eu(III) complex $\left(10^{-6} \mathrm{M}\right)$ was measured by Model-SP2558 spectrometer of Acton Research in methanol at the Institute of Physics - Vietnam Academy of Science and Technology.

\subsection{Synthesis of AnPy ligand}

Step 1: Synthesis of AnPy1

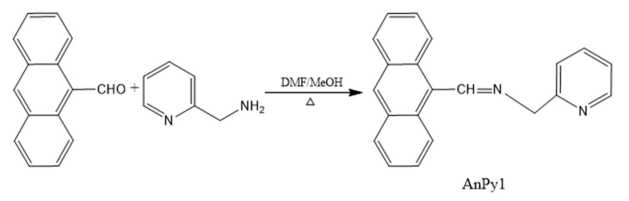

To a completely dissolved solution of anthracene-9-carboxaldehyde $(0.206 \mathrm{~g} ; 1$ $\mathrm{mmol})$ in $15 \mathrm{~mL}$ mixture $\mathrm{DMF} / \mathrm{MeOH}(\mathrm{v}: \mathrm{v}=1$ : 5) was added 2-picolylamine $(0.12 \mathrm{~mL} ; 1.1$ $\mathrm{mmol}$ ) and the solution was allowed to react for 36 hours at room temperature with constant stirring. The compound was not isolated and directly used for the next step.

Step 2: Synthesis of AnPy ligand

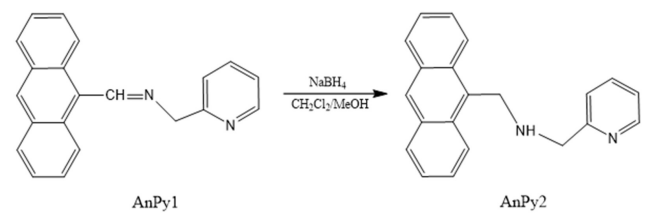

The Schiff base formed was reduced by adding solid $\mathrm{NaBH}_{4}(0.2 \mathrm{~g})$ to AnPyl. The mixture was further refluxed for 4 hours to ensure a complete reduction of the imine. After removal of the solvent, the resulting paleyellow solid was washed with plenty of water for 60 minutes and extracted with $\mathrm{CH}_{2} \mathrm{Cl}_{2}$ (20 $\mathrm{mL})$. The organic layer was evaporated to dryness where upon AnPy was obtained as a brown semisolid. (Yield: 70\%).

\subsection{Synthesis of $\mathrm{Eu}(\mathrm{TFPB})_{3}\left(\mathrm{H}_{2} \mathrm{O}\right)_{2}(\mathrm{Ml})$}

$\mathrm{Eu}_{2} \mathrm{O}_{3}(0.0704 \mathrm{~g}, 0.2 \mathrm{mmol})$ was dissolved in $\mathrm{HCl}$ at $150^{\circ} \mathrm{C}$, then distilled water was added and heated at $100^{\circ} \mathrm{C}$. A solution of $\mathrm{NaOH}$ $(0.048 \mathrm{~g}, 1.2 \mathrm{mmol})$ and HTFPB (0.2592 g, 1.2 $\mathrm{mmol})$ in $\mathrm{MeOH}(15 \mathrm{~mL})$ was added drop-wise under stirring to a solution in $\mathrm{MeOH}(15 \mathrm{~mL})$. The mixture was stirred at room temperature to afford a white solid. The product was washed by a large amount of $\mathrm{CCl}_{4}$ and air-dried [6]. Yield: $88 \%$. The organic layer was evaporated to dryness where upon AnPy was obtained as a brown semisolid. (Yield: 70\%).

\subsection{Synthesis of $E u(T F P B)_{3} A n P y(M 2)$}

A solution of AnPy $(1 \mathrm{mmol})$ in $\mathrm{CH}_{2} \mathrm{Cl}_{2}$ $(15 \mathrm{~mL})$ was added drop-wise under stirring to a solution of $\mathrm{M} 1$ in $\mathrm{EtOH}(15 \mathrm{~mL})$. The mixture was stirred at room temperature about 1 hour until a yellowish precipitate appeared. The solvent was removed in vacuum and the 
resulting solid was then washed with n-hexane. The titled compound was obtained as a paleyellow powder. which could be futher purified by recrystallization in EtOH and $\mathrm{CH}_{2} \mathrm{Cl}_{2}$ (v:v $=$ 1:1). Yield: $74 \%$.

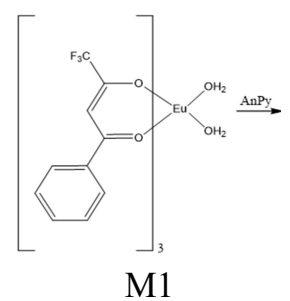

\section{Results and Discussion}

\subsection{Infrared spectroscopy}

The infrared spectrum of the complex $\mathrm{Eu}(\mathrm{TFPB})_{3} \mathrm{AnPy}$ is shown in Figure 1. Typical absorption bands of the complexes and ligand are shown in Table 1.

The IR spectrum of M1 exhibits the typical broad absorption in the region $3000 \div 3500 \mathrm{~cm}^{-}$ 1 , proposing the presence of water molecules, coordinated to the ion $\mathrm{Eu}^{3+}$. In contrast, the absence of the broad bands in the region $3000 \div$ $3500 \mathrm{~cm}^{-1}$ for M2 suggests that water molecules in M1 have been displaced by the nitrogen donors of AnPy ligand. The absorption at 1600 $\mathrm{cm}^{-1}$, which is typical for $\mathrm{C}=\mathrm{O}$ stretch in the HTFPB ligand is bathochromically shifted to $1610 \mathrm{~cm}^{-1}$ in M2 [6].

The absorption at $1081 \mathrm{~cm}^{-1}$ is assigned for $\mathrm{C}-\mathrm{N}$ stretch in AnPy ligand. In addition, the absorption band at $526 \mathrm{~cm}^{-1}$ which is attributable to Eu-N stretch in M2 confirms the complexation of $\mathrm{Eu}^{3+}$ ion with AnPy ligand through nitrogen atoms. The change in absorption frequency of $v_{\mathrm{C}=\mathrm{O}}$ compared with that in free ligand and the appearance of $v_{\mathrm{Eu}-\mathrm{N}}$ absorption in the low frequency proved HTFPB and AnPy ligands were coordinated with $\mathrm{Eu}(\mathrm{III})$.

\subsection{Single-crystal X-ray diffraction}

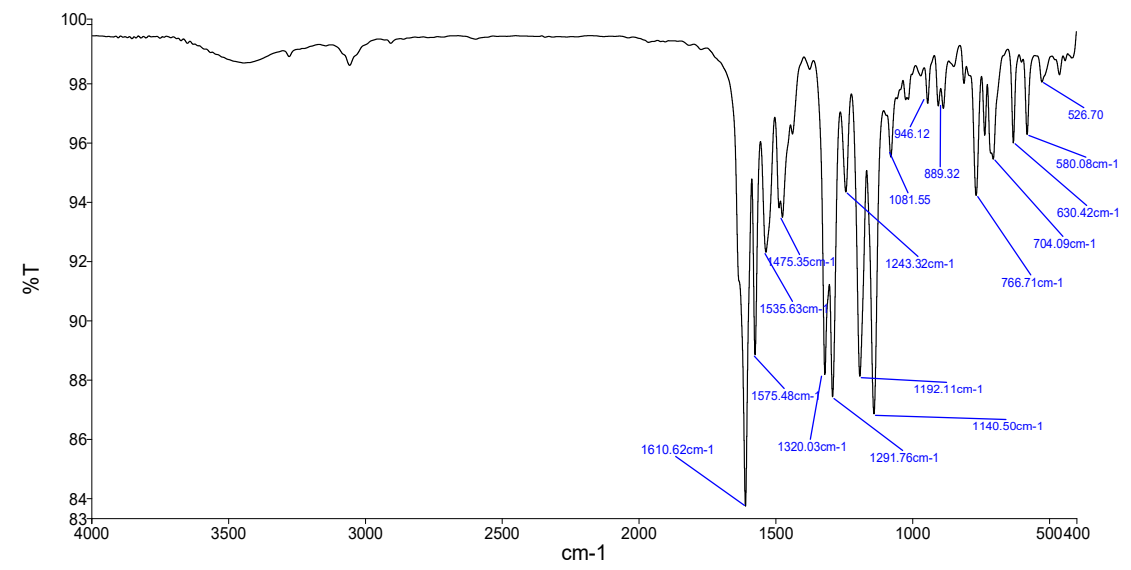

Fig. 1. The infrared spectrum of M2.

Table 1. Typical absorption bands in the infrared spectra of the complexes and ligand $\left(v, \mathrm{~cm}^{-1}\right)$

\begin{tabular}{c|c|c|c}
\hline Compounds & $v_{\mathrm{O}-\mathrm{H}}$ & $v_{\mathrm{C}-\mathrm{N}}$ & $v_{\mathrm{C}=\mathrm{O}}$ \\
\hline HTFPB & 3450 & - & 1600 \\
\hline M1 & 3400 & - & 1614 \\
\hline M2 & - & 1081 & 1610 \\
\hline
\end{tabular}


The structure of M2 was determined by single crystal X-ray diffraction (Figure 2). Crystal data and data collection parameters for the complex are given in Table 2. Selected bond lengths and angles are provided in Table 3. for $\mathrm{M} 2$.

Table 2. Crystal data and structure refinement

\begin{tabular}{cc}
\hline Formula & $\mathrm{C}_{51} \mathrm{EuF}_{9} \mathrm{~N}_{2} \mathrm{O}_{6} \mathrm{H}_{0.13}$ \\
\hline $\mathrm{M}_{\mathrm{w}} / \mathrm{g} \cdot \mathrm{mol}^{-1}$ & 1059.62 \\
\hline $\mathrm{Crystal}$ system & Monoclinic \\
\hline $\mathrm{a} / \AA$ & $39.111(9)$ \\
\hline $\mathrm{b} / \AA$ & $10.777(3)$ \\
\hline $\mathrm{c} / \AA$ & $26.036(6)$ \\
\hline$\alpha /^{\circ}$ & 90 \\
\hline$\beta /{ }^{\circ}$ & $116.895(4)$ \\
\hline$\gamma /{ }^{\circ}$ & 90 \\
\hline Volume $/ \AA^{3}$ & $9787(4)$ \\
\hline Space group & $\mathrm{C} 2 / \mathrm{c}$ \\
\hline $\mathrm{Z}$ & 8 \\
\hline $\mathrm{R} 1 / w R 2[\mathrm{I}>=2 \sigma(\mathrm{I})]$ & $0.0865 / 0.1886$
\end{tabular}

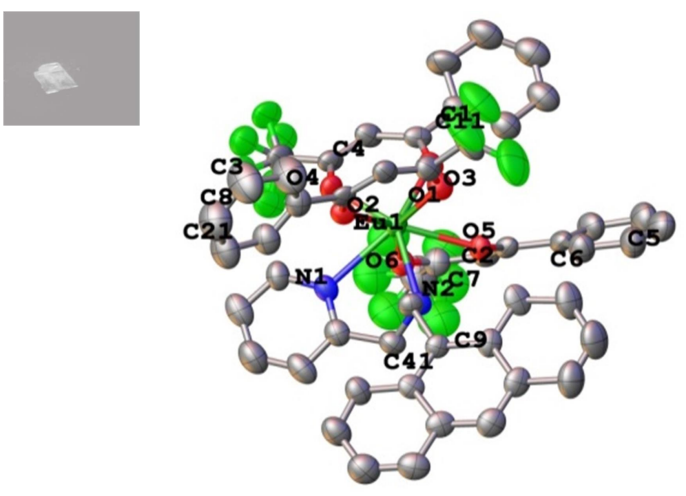

Fig 2. Molecular structure of compound M2 solid state. Color scheme: Eu, blue; N, pale blue; O, red; $\mathrm{F}$, green; C, gray.

Table 3. Selected bond lengths $(\AA)$ and angles $\left({ }^{\circ}\right)$ for M2.

\begin{tabular}{cccc}
\hline \multicolumn{4}{c}{ Bond lengths $(\AA)$ for M2 } \\
\hline Eu1-O1 & $2.366(13)$ & O4-C4 & $1.27(2)$ \\
\hline Eu1-O2 & $2.361(12)$ & O6-C9 & $1.27(2)$ \\
\hline Eu1-O3 & $2.390(12)$ & O2-C3 & $1.28(2)$ \\
\hline Eu1-O4 & $2.344(11)$ & N1-C11 & $1.35(2)$ \\
\hline Eu1-O5 & $2.386(12)$ & C21-N2 & $1.52(2)$
\end{tabular}

\begin{tabular}{cccc} 
Eu1-O6 & $2.366(13)$ & $\mathrm{C} 11-\mathrm{C} 21$ & $1.52(3)$ \\
\hline Eu1-N1 & $2.622(15)$ & $\mathrm{C} 1-\mathrm{C} 2$ & $1.37(3)$ \\
\hline Eu1-N2 & $2.619(14)$ & $\mathrm{C} 2-\mathrm{C} 3$ & $1.42(3)$ \\
\hline $\mathrm{O} 1-\mathrm{C} 1$ & $1.28(2)$ & $\mathrm{C} 9-\mathrm{C} 8$ & $1.36(3)$ \\
\hline O3-C6 & $1.256(19)$ & $\mathrm{C} 7-\mathrm{C} 8$ & $1.44(3)$ \\
\hline O5-C7 & $1.295(19)$ \\
\hline \multicolumn{3}{c}{ Bond angles $\left(^{0}\right)$ for M2 } \\
\hline O1-Eu1-O2 & $70.6(4)$ \\
\hline O1-Eu1-O3 & $81.0(4)$ \\
\hline O4-Eu1-O3 & $72.4(4)$ \\
\hline O6-Eu1-O5 & $70.5(4)$ \\
\hline N1-Eu1-N2 & $64.6(5)$ \\
\hline N1-C11-C21 & $114.4(18)$ \\
\hline C11-C21-N2 & $110.8(16)$ \\
\hline
\end{tabular}

The structure of the complex reveals a coordination number eight of central metal ion, in which $\mathrm{Eu}^{3+}$ is bonded to six oxygen atoms from three TFPB and two nitrogen atoms from the AnPy ligand. The bond lengths of Eu1-O are $2.344 \div 2.390 \AA$. The bond lengths of $\mathrm{Eu}(\mathrm{III})$ with two nitrogen atoms of AnPy are $2.622 \AA$ and $2.619 \AA$. The O-Eu1-O bond angles are nearly the same and in the range of $70.6 \div 81^{\circ}$, longer than that of $\mathrm{N}-\mathrm{Eu} 1-\mathrm{N}$ $\left(64.6^{0}\right)$. The $\mathrm{C}-\mathrm{C}$ bond length in the diketone of $\mathrm{M} 2$ is $1.36 \div 1.52 \AA$, shorter than the $\mathrm{C}-\mathrm{C}$ bond length $(1.54 \AA)$ but longer than that of $\mathrm{C}=\mathrm{C}$ (1.34 $\AA$ ). Similarly, the $\mathrm{C}-\mathrm{O}$ bond length in the diketone of $\mathrm{M} 2$ is $1.256 \div 1.295 \AA$ is also shorter than the length of the $\mathrm{C}-\mathrm{O}$ but longer than the bond length of $\mathrm{C}=\mathrm{O}$. This confirms the delocalization of $\pi$ electrons in the $\beta$-diketonate upon $\mathrm{Eu}(\mathrm{III})$ and TFPB ligand complexation. The coordination of Eu(III) with AnPy ligand through two nitrogen atoms forms a fivemembered chelate ring. Notably, aliphatic nitrogen is deviated from pyridine ring plane by a distance of $0.883 \AA$.

\subsection{Absorption Spectra}

Reaction of $\mathrm{M} 2$ with ${ }^{1} \mathrm{O}_{2}$ is given by this scheme:

To determine the luminescent properties of $\beta$-diketonate with rare earth metal, we measured the absorption spectrum of the ligand and $\mathrm{Eu}(\mathrm{III})$ complexes in the region of $200 \div$ $800 \mathrm{~nm}$ in $\mathrm{CH}_{2} \mathrm{Cl}_{2}$ solvent. 
The absorption spectra of M2 and AnPy are displayed in Fig 3.
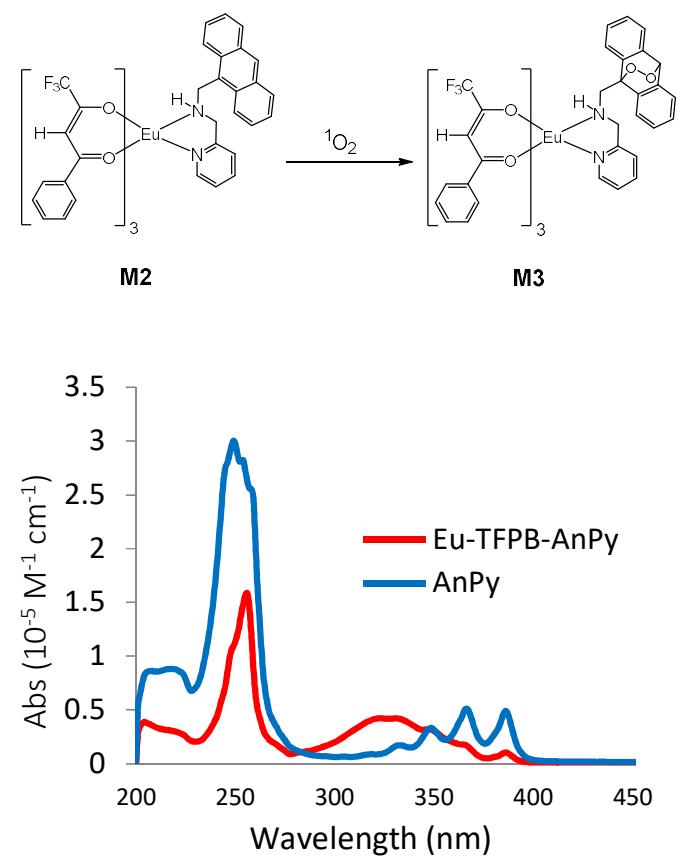

Fig 3. Absorption spectra of $\mathrm{M} 2, \mathrm{AnPy}$ in $\mathrm{CH}_{2} \mathrm{Cl}_{2}$ solvent at room temperature.

AnPy ligand and M2 complexes exhibit strong absorption in the region $200 \div 400 \mathrm{~nm}$. The broad bands observed at 336 and $337 \mathrm{~nm}$ in the spectrum of M2 are ascribable to singletsinglet $\pi-\pi^{*}$ enolic transition arising from $\beta$ diketonate fragment. These absorption bands are shifted slightly on the long-wave region compared with its position in HTFPB free (334 $\mathrm{nm})$, suggesting the perturbation of the $\mathrm{Eu}^{3+}$ upon complexation. The bands at lower wavelength around 300-400 $\mathrm{nm}$ are anthracenecentered $\pi-\pi^{*}$ transition. The auxiliary ligand AnPy is also absorbed at ultraviolet wavelengths $265-305 \mathrm{~nm}$. The lanthanide $\mathrm{f}-\mathrm{f}$ transitions are "Laporte-forbidden", therefore unable to be observed in the absorption spectrum of the complex.

\subsection{Photoluminescence spectroscopy}

The photoluminescence spectra of M1, M2, $\left[\mathrm{Eu}(\mathrm{TFPB})_{3}(\mathrm{EP}-\mathrm{AnPy})\right] \quad(\mathrm{M} 3)$ were characterized in the solution $10^{-5} \mathrm{M}$ at $\lambda_{\mathrm{kt}}=330$ $\mathrm{nm}$ and shown in the Figure 4.

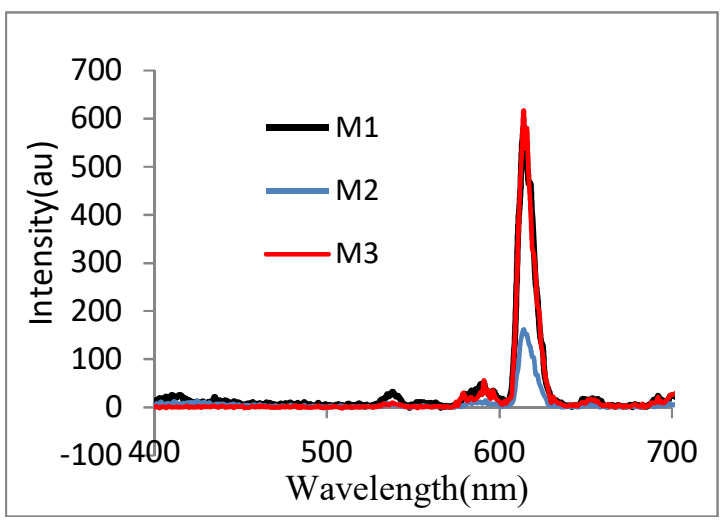

Fig 4. PL spectra of M1, M2, M3.

The results indicate that the M1 complex emits red luminescence, M2 gives no emission and M3 emits red luminescence. The emission lines at $540,592,614$ và $704 \mathrm{~nm}$ are assigned to the ${ }^{5} \mathrm{D}_{0} \rightarrow{ }^{7} \mathrm{~F}_{\mathrm{J}}(\mathrm{J}=0-4)$ transitions of $\mathrm{Eu}(\mathrm{III})$, the strongest transition ${ }^{5} \mathrm{D}_{0} \rightarrow{ }^{7} \mathrm{~F}_{2}$ centered at $614 \mathrm{~nm}$. The emission intensity of M1 and M3 is much stronger than that of M2. This emission turning-on property of M2 complex upon interaction with ${ }^{1} \mathrm{O}_{2}$ can be used to fabricate a luminescent sensor to detect small molecules in living organisms.

\section{Conclusion}

Europium(III) complexes containing benzoyltrifluoroacetone and (1-(anthracen-9yl)-N-(pyridine-2-ylmethyl) methane amine) ligands were successfully synthesized. The structures of these complexes were determined by various physical methods. The results confirmed that M2 reveal a coordination number of eight for central metal ion, in which $\mathrm{Eu}(\mathrm{III})$ are bonded to six oxygen atoms from three TFPB ligands and two nitrogen atoms from the AnPy ligand with R1 $=0.0865$. The $\mathrm{X}$-ray structures also reveal five-membered 
chelate ring of AnPy with $\mathrm{Eu}^{3+}$ ion in M2. The ancillary ligand AnPy has displace $\mathrm{H}_{2} \mathrm{O}$ molecules in the coordination bridge of M1. Luminescent properties of complexes have been determined by UV-Vis spectrophotometric method. The results show that M1 and M2 complexes exhibit strong absorption in the region $200 \div 400 \mathrm{~nm}$. The M2 complex has been initially investigated by the PL spectra. It showed that such a complex can be used as luminescence sensor through the on-off luminescence switching.

\section{Acknowledgments}

This work was completed with financial support from the Ministry of Education and Training of Vietnam, under the project B2018SPH- 49.

\section{References}

[1] B. Song, G. Wang, M. Tan, J. Yuan, A europium(III) complex as an efficient singlet oxygen luminescence probe, Journal of the
American Chemical Society. 128 (2006) 1344213450. https://doi.org/10.1021/ja062990f.

[2] Y. Wang, H. Wang, X. Zhao, J. Yuan, J. Wu, A $\beta$-diketonate-europium(III) complex-based fluorescent probe for highly sensitive time-gated luminescence detection of copper and sulfide ions in living cells", New J. Chem, 41 (2017) 59815987. https://doi.org/10.1039/C7NJ00802C.

[3] S.K. Pedersen, J. Holmehave, P.R. Ogilby, Aarhus Sensor Green: A Fluorescent Probe for Singlet Oxygen, J. Org. Chem, 79 (2014) 30793087. https://doi.org/10.1021/jo500219y.

[4] J. Yuasa, T. Nakagawa and T. Kawai, Photoactivatable europium luminescence turnonby photo-oxygenation of $\beta$-diketone having pyrrole rings, Chem. Commun, 53 (2017) 67486751. https://doi.org/10.1039/C7CC03753H

[5] H.J. Yin, Y.J. Liu, J. Gao, K.Z. Wang, A highly sensitive and selective visible-light excitable luminescent probe for singlet oxygen based on a dinuclear ruthenium complex, Dalton Trans, 46 (2017) 3325-3331. https://doi.org/10.1039/C6DT 04813G.

[6] T. T. Nguyet, D. T. Hien, N. H. Huy, Ulrich Abram and N. M. Hai, Novel lanthanide(III) ternary complexes with naphthoyltrifluoroace tone: A synthetic and spectroscopic study, Zeitschrift für anorganische und allgemeine Chemie, 641 (2015) 1934-1940. https://doi.org /10.1002/zaac.201500158. 